\title{
Blendas PHB/Copoliésteres Biodegradáveis - Biodegradação em Solo
}

\author{
Suzan A. Casarin, José A. M. Agnelli \\ Departamento de Engenharia de Materiais, UFSCar \\ Sônia M. Malmonge \\ Centro de Engenharia, Modelagem e Ciências Sociais Aplicadas, UFABC \\ Francisco Rosário \\ Curso de Engenharia de Materiais, UTFPR
}

\begin{abstract}
Resumo: Este trabalho apresenta os resultados do comportamento de blendas do polímero biodegradável PHB poli(hidroxibutirato) com os copoliésteres também biodegradáveis EastarBio ${ }^{\circledR}$ e Ecoflex ${ }^{\circledR}$, na composição de $75 \%$ de PHB e $25 \%$ dos copoliésteres, em contato com solo composto simulado. Foi também avaliada a influência da adição de pó de serra ou farinha de madeira, na proporção de $70 \%$ da blenda e 30\% de pó de serra (p.d.s.). A biodegradação foi avaliada para amostras após 30, 60 e 90 dias em contato com solo, através de análises gravimétricas, morfológicas e mecânicas. A preparação inicial dos grânulos dos compostos poliméricos foi feita por extrusão, utilizando uma extrusora dupla-rosca e a moldagem dos corpos de prova foi realizada através da moldagem por injeção. Os materiais estudados biodegradam nas condições testadas. A blenda PHB/EastarBio ${ }^{\circledR}(75 / 25)+30 \%$ p.d.s. apresentou maior redução de massa, $29 \%$ após 90 dias. Notou-se que a biodegradação se inicia pela superfície do material e que 90 dias são insuficientes para observar alterações internas.
\end{abstract}

Palavras-chave: Polímeros biodegradáveis, blendas biodegradáveis, poli(hidroxibutirato) - PHB, EastarBio ${ }^{\circledR}$, Ecoflex $^{\circledR}$.

\section{Biodegradable PHB/Copolyester Blends - Biodegradation in Soil}

Abstract: This paper reports on blends made with the biodegradable polymers poly(hydroxybutyrate) (PHB) and Eastar Bio ${ }^{\circledR}$ or Ecoflex ${ }^{\circledR}$ copolyesters, in contact with simulated compound soil. The blends had $75 \%$ of PHB and $25 \%$ of copolyesters. We also analyzed the influence from adding $30 \%$ of powder-wood or wood flour (WPC) to $70 \%$ of the blend. Biodegradation was analyzed for samples after 30, 60 and 90 days in contact with soil, through thermogravimetric, morphological and mechanical analyses. The initial preparation of the granules of polymeric compounds was made by extrusion, using a twin-screw extruder and the molding of the specimens was performed by injection molding. The analysis indicated material biodegradation under the conditions tested. The PHB/Eastar Bio ${ }^{\circledR}$ blend $(75 / 25)+30 \%$ WPC exhibited the highest degradation with $29 \%$ of mass loss at the end of 90 days. Biodegradation started on the material surface and 90 days were not sufficient to induce noticeable internal changes.

Keywords: Biodegradable polymers, biodegradable blends, poly(hydroxybutyrate) - PHB, Eastar Bio ${ }^{\circledR}$, Ecoflex $^{\circledR}$.

\section{Introdução}

Desde o início do século passado, o uso de plásticos tem se tornado cada vez mais frequente na sociedade. Basta olhar ao redor para se perceber a incrível quantidade de produtos produzidos pelo homem que utiliza polímeros como matéria-prima ${ }^{[1]}$. Isso se deve às características como: durabilidade, atoxicidade, baixo custo, facilidade para serem moldados, capacidade de serem reciclados e versatilidade de aplicação ${ }^{[2]}$.

Os produtos que empregam polímeros sintéticos convencionais como matéria prima são considerados inertes ao ataque imediato de microrganismos. Isso faz com que esses produtos apresentem um maior ciclo de vida útil e, consequentemente, acarretem sérios problemas ambientais, visto que, após o seu descarte, contribuem para o aumento da quantidade de resíduos plásticos no meio ambiente $^{[3]}$.

A preocupação com o meio ambiente tem se tornado algo concreto nos dias atuais, gerando uma busca incessante de tecnologias que resultem em produtos e subprodutos obedecendo a um ciclo fechado sustentável ${ }^{[4]}$. A substituição de parte dos polímeros sintéticos por polímeros biodegradáveis constitui uma alternativa viável, uma vez que existe a possibilidade de misturar polímeros de naturezas diferentes, na forma de blendas, garantindo a sua aplicabilidade e, com isso, melhorar a biodegradabilidade do material resultante ${ }^{[2]}$.
Os principais polímeros biodegradáveis são os poliésteres baseados nos ácidos hidróxi-carbônicos. Os poliésteres biodegradáveis ainda apresentam um custo elevado, mas já despertam grande interesse pelo fato de serem produzidos por fermentação ou rotas sintéticas acessíveis ${ }^{[5,6]}$. Entre os polímeros biodegradáveis mais conhecidos, destacam-se o poli(hidroxibutirato)- $\mathrm{PHB}^{[2,6-10]}$, a poli( $\varepsilon$-caprolactona)- $\mathrm{PCL}^{[10,11]}$, o poli(ácido láctico) - PLA ${ }^{[11-13]}$ e copoliésteres biodegradáveis (Ecoflex ${ }^{\circledR[6,14]}$ da BASF, e Eastar Bio ${ }^{\circledR[15,16]}$ da Eastaman Chemical Company). Estes polímeros apresentam excelentes aplicações no setor de embalagens e filmes, devido a sua alta resistência à umidade, à gordura, à mudança de temperatura e propriedade de barreira a gases. Eles também são potenciais matérias primas para uso como biomateriais, no desenvolvimento de dispositivos de osteosíntese, suturas, implantes e liberação controlada de ativos ${ }^{[6]}$.

O PHB é um termoplástico duro e quebradiço, escoa facilmente durante o processamento, não é solúvel em água e é pouco permeável a $\mathrm{O}_{2}, \mathrm{H}_{2} \mathrm{O}$ e $\mathrm{CO}_{2}$. O PHB é totalmente isotático e capaz de atingir um grau de cristalinidade na faixa de 55-80\%. A temperatura de transição vítrea (Tg) do PHB é de aproximadamente $5{ }^{\circ} \mathrm{C}$ e a temperatura de fusão (Tm), de $175^{\circ} \mathrm{C}$. A maior desvantagem do PHB é sua baixa estabilidade térmica: acima de $170{ }^{\circ} \mathrm{C}$ ocorre diminuição na massa molar ${ }^{[7]}$. 
No Brasil, a produção de PHB foi desenvolvida por uma joint venture entre a Copersucar (Cooperativa dos Produtores de Cana-de-açúcar do Estado de São Paulo), o IPT (Instituto de Pesquisas Tecnológicas) e pelo ICB (Instituto de Ciências Biomédicas da USP) ${ }^{[17,18]}$. A produção de PHB pela PHB Industrial S/A é a única produção industrial de $\mathrm{PHB}$ a partir de cana-de-açúcar e integrada em usina sucroalcooleira. Essa empresa opera desde 2005 com uma planta de capacidade máxima de 60 toneladas de PHB por ano. Enquanto na Europa, o PHB é produzido a US\$10-20,00/kg, no Brasil esses custos estão entre US\$2,5-5,00/kg ${ }^{[19]}$. Após o consumo, o PHB pode ser descartado em aterros sanitários sem causar impacto ao meio ambiente, podendo ser biodegradado por bactérias e fungos ${ }^{[20]}$, restando apenas água, gás carbônico e biomassa ${ }^{[6]}$.
O Ecoflex ${ }^{\circledR}$ é um polímero sintético biodegradável produzido pela empresa alemã Basf, que em parceria com a Naturework lançou no mercado o Ecovio ${ }^{\circledR}$, blenda Ecoflex ${ }^{\circledR} /$ PLA. Esta blenda pode ser utilizada na produção de embalagens, filmes, tubetes para reflorestamento, sacolas plásticas e embalagens para cosméticos ${ }^{[21]}$. Os poliésteres alifáticos aromáticos possuem capacidade instalada em torno de 30.000 t/ano com destaque para os polímeros Bionelle (Showa, Japonesa), Eastar Bio ${ }^{\circledR}$ (Eastman, Americana) e Ecoflex ${ }^{\circledR}$ (Basf, Alemã $)^{[6]}$.

As vantagens da utilização de reforços lignocelulósicos em polímeros são baixa densidade, baixa abrasividade, possibilidade de incorporação de elevados teores resultando em elevada rigidez, manutenção da reciclabilidade, biodegradabilidade e grande variedade de cargas reforçativas existentes ${ }^{[7]}$.

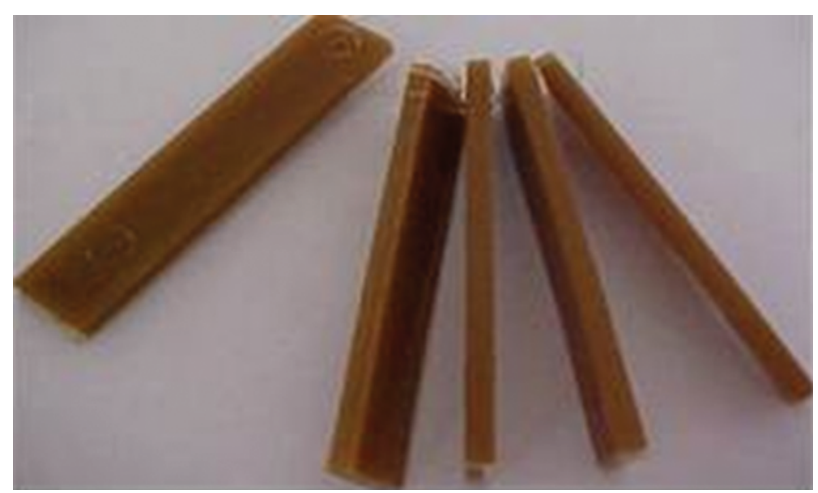

(a)

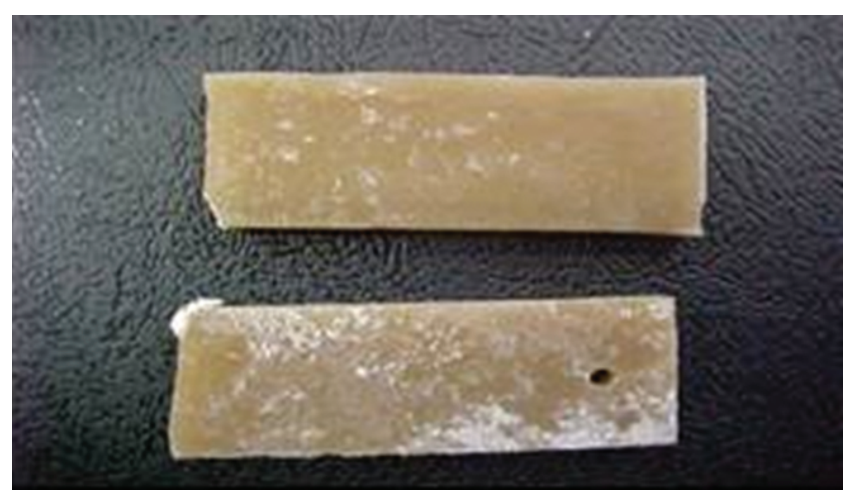

(b)

Figura 1. Corpos de prova do PHB puro (a) antes e (b) após 90 dias em contato com o solo.

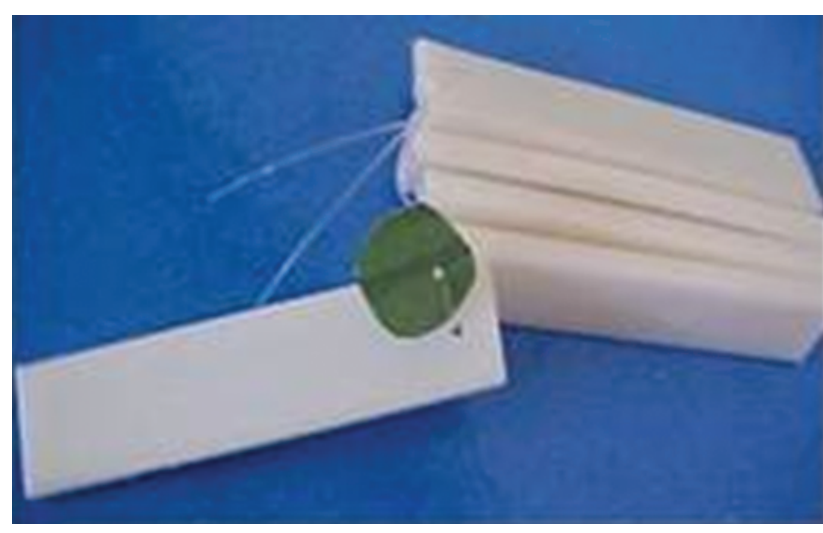

(a)

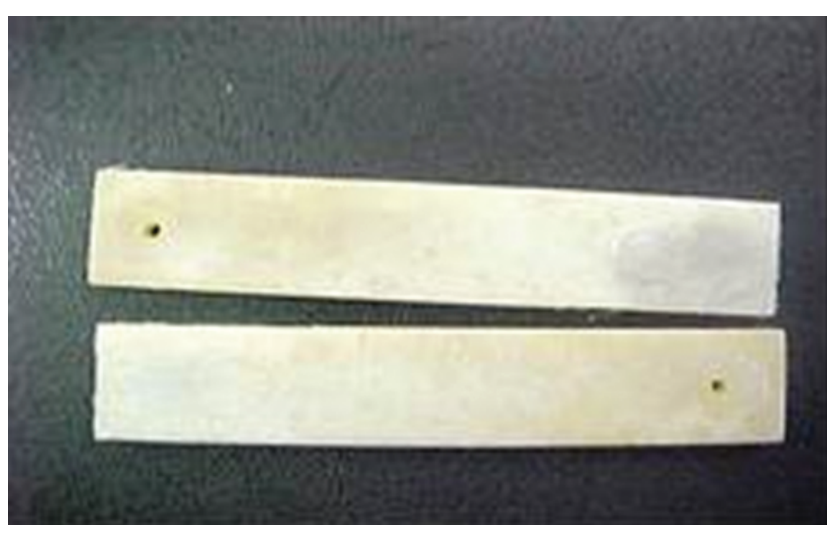

(b)

Figura 2. Corpos de prova do copoliéster Eastar Bio $^{\circledR}$ puro (a) antes e (b) após 90 dias em contato com o solo.

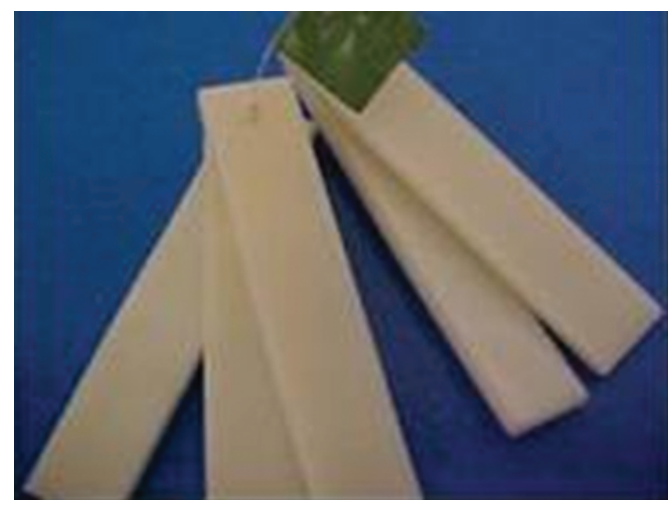

(a)

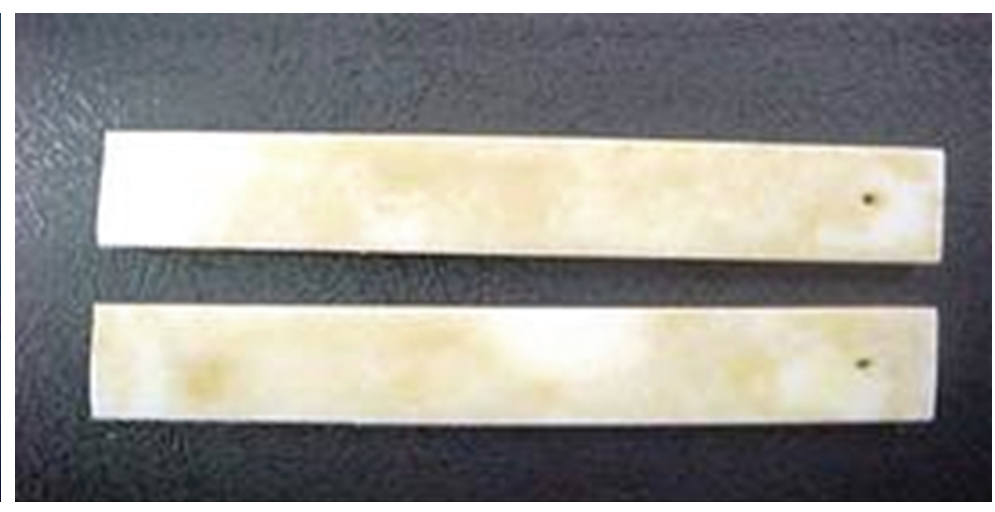

(b)

Figura 3. Corpos de prova do copoliéster Ecoflex ${ }^{\circledR}$ puro (a) antes e (b) após 90 dias em contato com o solo. 
Desta maneira, objetivou-se neste trabalho o desenvolvimento de blendas de PHB/Copoliésteres (75/25) com a intenção de obter-se uma redução na rigidez da matriz do PHB. Realizou-se também um estudo da incorporação de pó de serra nas blendas PHB/Ecoflex ${ }^{\circledR} \mathrm{e}$ PHB/Eastar Bio ${ }^{\circledR}$, visando à obtenção de um material para aplicação em curto prazo, a um custo viável, com propriedades físico-mecânicas adequadas e apresentando biodegradação, a biodegradabilidade dos materiais estudados foi acompanhada por 30, 60 e 90 dias por análise visual, gravimétrica, morfológica e mecânica.

\section{Material e Métodos}

Para a realização deste estudo foram preparadas amostras de blendas biodegradáveis utilizando o $\mathrm{PHB}$, fornecido pela $\mathrm{PHB}$ Industrial S/A e dois copoliésteres com propriedades térmicas e mecânicas semelhantes, mas de fornecedores diferentes: o copoliéster biodegradável Eastar Bio $^{\circledR}$ proveniente da Eastman Chemical Company e o copoliéster biodegradável Ecoflex ${ }^{\circledR}$ proveniente da BASF. As blendas PHB/Eastar Bio ${ }^{\circledR}$ e PHB/Ecoflex ${ }^{\circledR}$ foram preparadas nas seguintes composições (100/0, 75/25, 0/100), sendo que as blendas $\mathrm{PHB} /$ copoliésteres $(75 / 25)$ foram modificadas com a incorporação de $30 \%$ de pó de serra, com granulometria de malha 100, fornecido pela Pinhopó Moagem de Madeiras Ltda.

\section{Processamento dos materiais}

Os materiais PHB e pó de serra foram previamente secos em estufa com circulação de ar a uma temperatura de aproximadamente
$60{ }^{\circ} \mathrm{C}$ por 24 horas. Para os copoliésteres Eastar Bio $^{\circledR}$ e Ecoflex ${ }^{\circledR}$ foi utilizado uma temperatura de aproximadamente $50{ }^{\circ} \mathrm{C}$ por 4 horas.

A preparação inicial dos grânulos das composições poliméricas foi feita por extrusão e a moldagem dos corpos de prova foi realizada através da moldagem por injeção. Para a extrusão das blendas poliméricas foi empregada uma extrusora de dupla rosca, modular, interpenetrante, co-rotacional, modelo ZSK 30 (diâmetro $\mathrm{D}=30 \mathrm{~mm}$ e $(\mathrm{L} / \mathrm{D})=35)$, da marca Werner \& Pfleiderer, com um perfil de rosca adequado para a incorporação de elevados teores de carga particulada em matrizes poliméricas termoplásticas.

Para a preparação da blenda PHB/Ecoflex ${ }^{\circledR}(75 / 25)$, os polímeros PHB e o copoliéster Ecoflex ${ }^{\circledR}$, em grânulos foram introduzidos no funil de alimentação da extrusora dupla rosca, sendo que a sua dosagem foi controlada por um alimentador vibratório (alimentador específico para polímeros na forma de grânulos). Para a preparação da blenda modificada, o pó de serra foi introduzido em um dos funis de alimentação (alimentador específico para materiais em forma de pó) e a blenda PHB/Ecoflex ${ }^{\circledR}(75 / 25)$ foi introduzida em outro funil de alimentação. Os mesmos procedimentos foram seguidos para a preparação da blenda PHB/Eastar Bio ${ }^{\circledR}(75 / 25)$ e $70 \%$ PHB/Eastar Bio $^{\circledR}(75 / 25) / 30 \%$ pó de serra. Após a extrusão, todos os materiais foram granulados. $\mathrm{Na}$ injeção dos polímeros e suas blendas utilizou-se uma injetora Arburg Allorunder modelo 270V/300-120, completamente automatizada, com capacidade de injeção de $54 \mathrm{~cm}^{3}$ e diâmetro de rosca de $25 \mathrm{~mm}$.

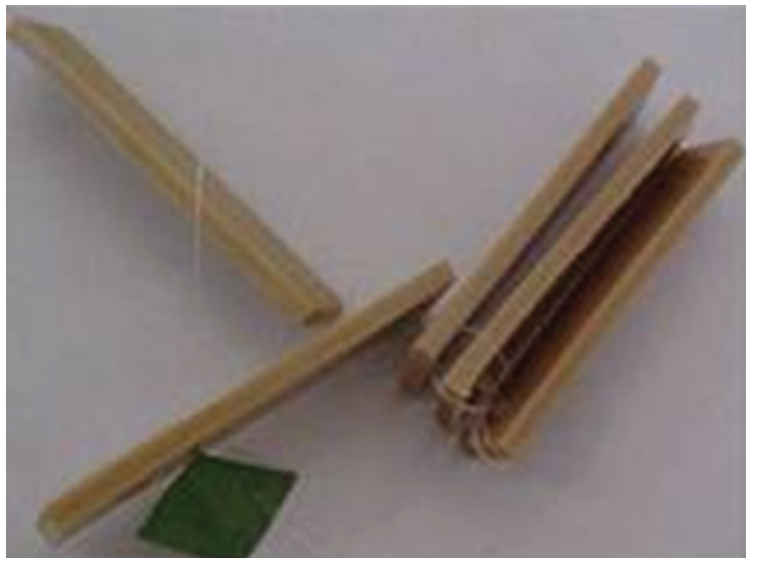

(a)

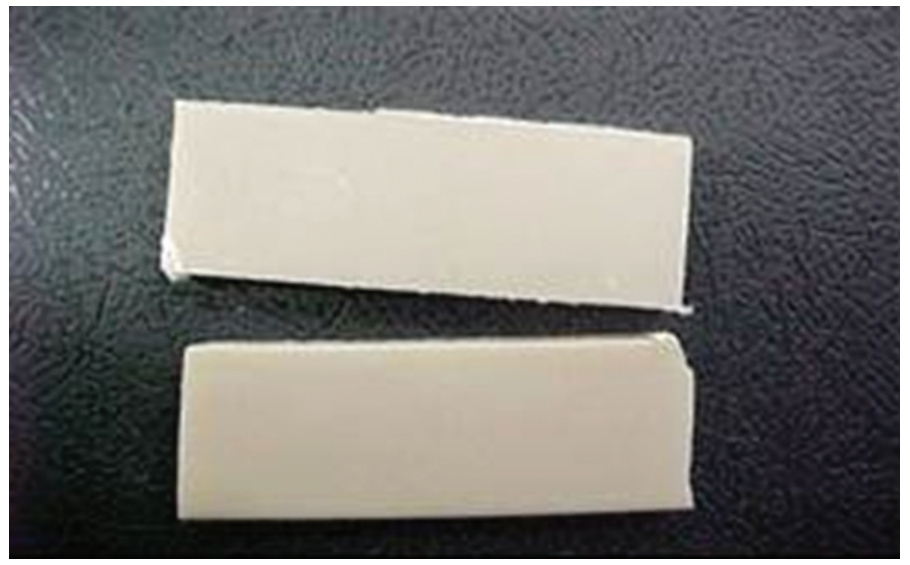

(b)

Figura 4. Corpos de prova da blenda PHB/Ecoflex ${ }^{\circledR}$ (75/25) (a) antes e (b) após 90 dias em contato com o solo.

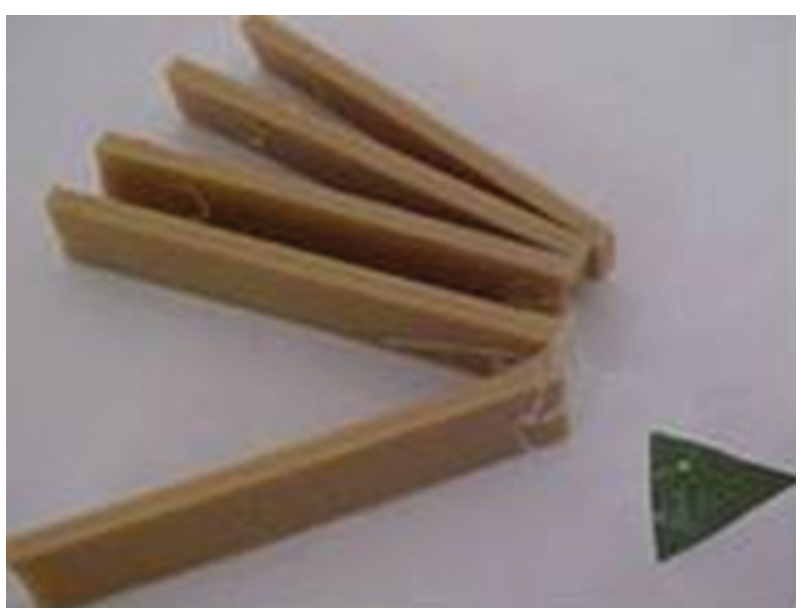

(a)

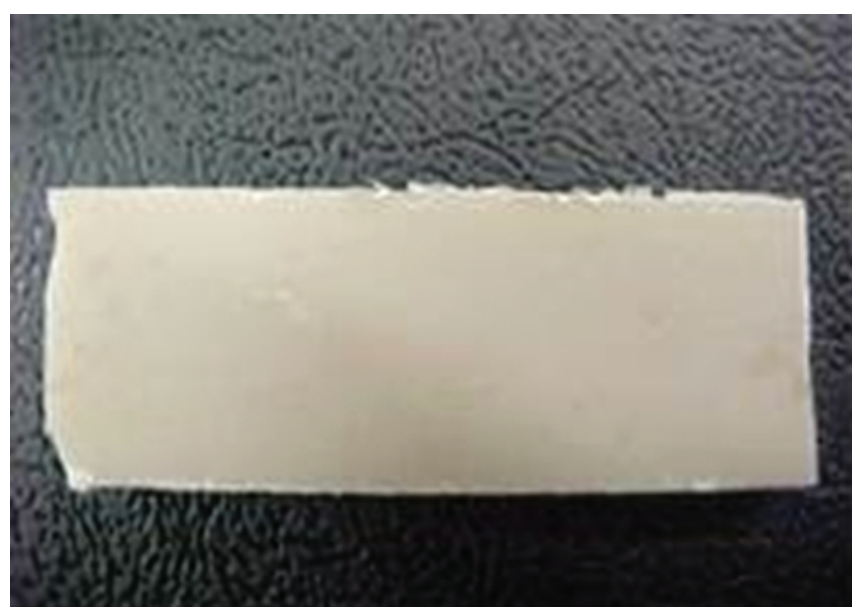

(b)

Figura 5. Corpos de prova da blenda PHB/Eastar Bio ${ }^{\circledR}(75 / 25)$ (a) antes e (b) após 90 dias em contato com o solo. 


\section{Biodegradação em solo}

O ensaio de biodegradação em solo foi realizado seguindo as normas ASTM D6003 e ASTM G160 ${ }^{[22,23]}$. O solo (Fertilizante orgânico composto) utilizado foi proveniente da Provaso Indústria e Comércio de Fertilizantes Orgânicos Ltda, com as seguintes especificações: N (mínimo) 1\%; Matéria Orgânica mínima 40\%; Umidade máxima 40\%; pH 6; C/N máximo 18/1.

As amostras foram colocadas em contato com solo durante 30, 60 e 90 dias, sendo que cada conjunto continha 5 corpos de prova de cada composição: PHB puro, Ecoflex ${ }^{\circledR}$ puro, Eastar Bio ${ }^{\circledR}$ puro, as blendas PHB/Ecoflex ${ }^{\circledR}(75 / 25)$ e PHB/Eastar Bio ${ }^{\circledR}(75 / 25)$ e as blendas modificadas PHB/Ecoflex ${ }^{\circledR}(75 / 25) /$ pó de serra $(70 / 30)$ e PHB/Eastar Bio ${ }^{\circledR}(75 / 25)$ /pó de serra (70/30). Foram usados corpos de prova injetados para ensaios de impacto seguindo as especificações da norma ASTM D-256 244$]$.

\section{Análise macroscópica}

Para a realização de uma análise visual macroscópica foram obtidas fotos de todos os corpos de prova das amostras em estudo, antes de entrarem em contato com o solo e ao término de cada período. Foram analisados cinco corpos de provas de cada amostra em estudo, sendo que os corpos de prova de cada amostra foram amarrados com um fio de nylon e identificados com símbolos.

\section{Análise gravimétrica}

Para a verificação da perda de massa, os corpos de prova de cada amostra foram pesados antes de serem submetidas ao contato com o solo e após o término de cada período, para obter assim a porcentagem de perda de massa das amostras ao longo do tempo de biodegradação.

\section{Análise por Microscopia Eletrônica de Varredura - MEV}

Essa análise foi utilizada para avaliar a morfologia do interior dos corpos de prova das blendas PHB/Ecoflex ${ }^{\circledR}(75 / 25)$, PHB/ Eastar Bio $^{\circledR}(75 / 25)$, PHB/Ecoflex ${ }^{\circledR}(75 / 25) /$ pó de serra $(70 / 30)$ e PHB/Eastar Bio $^{\circledR}(75 / 25) /$ pó de serra $(70 / 30)$, antes e após serem submetidas ao contato com solo.

A análise foi realizada no CCDM (Centro de Caracterização e Desenvolvimento de Materiais/UFSCar), utilizando um Microscópio Eletrônico de Varredura Stereoscan 440. Para a análise microscópica da fratura os corpos de prova foram fraturados criogenicamente em nitrogênio líquido e foram metalizados com ouro.

\section{Ensaio mecânico}

Para verificar alterações nas propriedades mecânicas ao longo do tempo em contato com o solo, foi realizado um ensaio mecânico de impacto. Os ensaios foram realizados para corpos de prova com entalhe na Máquina de Ensaios de Impacto Izod Ceast code 6545100, com um pêndulo de 2,0 J, seguindo a norma ASTM D-256 $6^{[24]}$. Para cada amostra foram ensaiados 5 corpos de prova e o resultado expresso como a média destes valores. O ensaio só não foi realizado para os copoliésteres puros, por não fraturarem nas condições de ensaio.

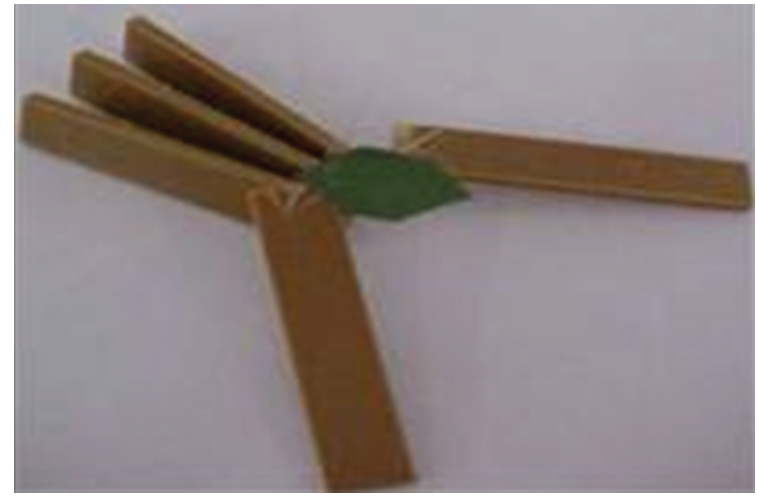

(a)

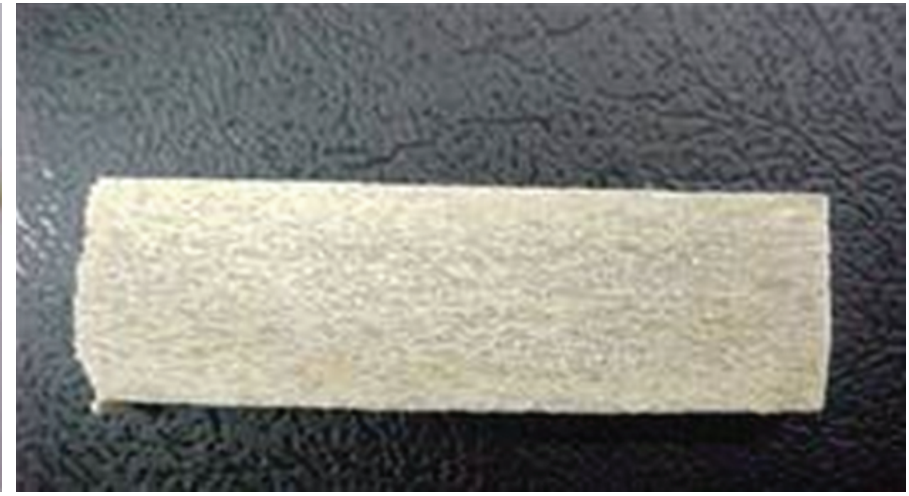

(b)

Figura 6. Corpos de prova da blenda PHB/Ecoflex ${ }^{\circledR}(75 / 25) /$ pó de serra (70/30) (a) antes e (b) após 90 dias em contato com o solo.

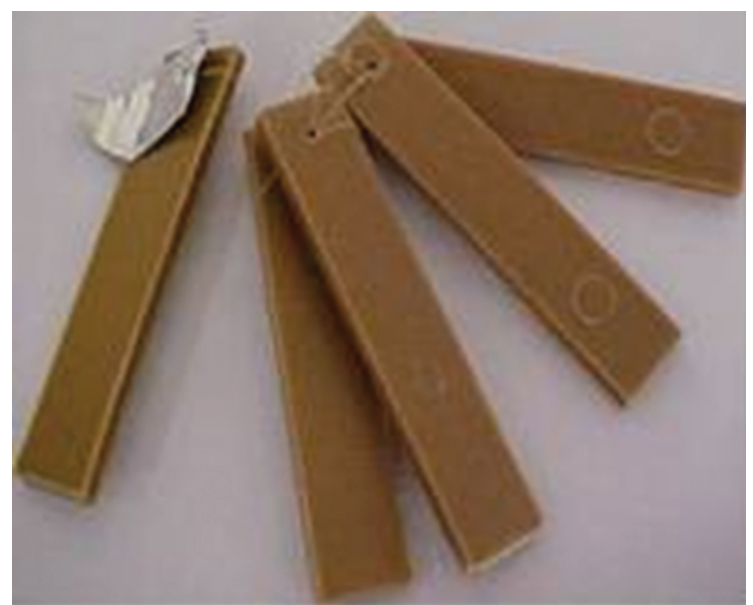

(a)

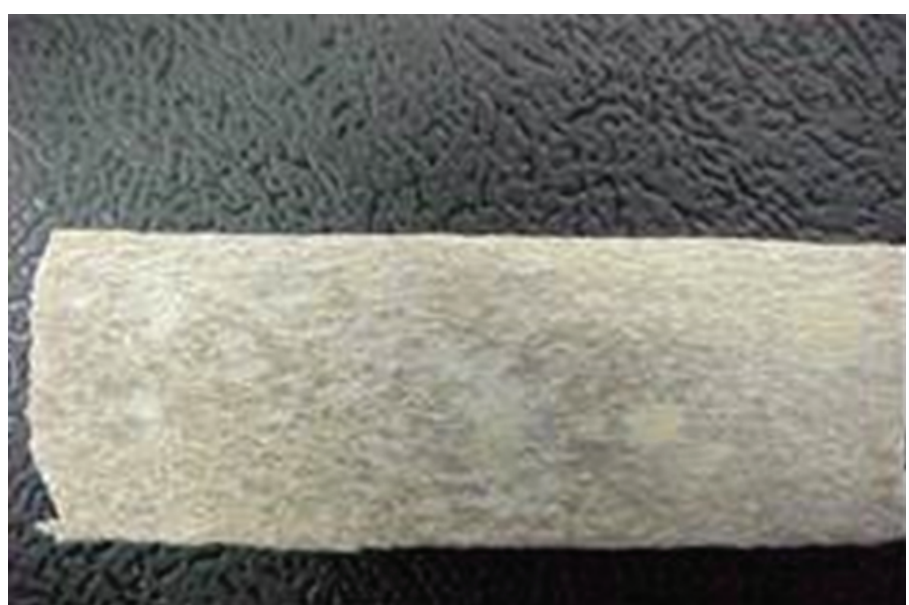

(b)

Figura 7. Corpos de prova da blenda PHB/Eastar Bio ${ }^{\circledR}$ (75/25)/pó de serra (70/30) (a) antes e (b) após 90 dias em contato com o solo. 


\section{Resultados e Discussões}

\section{Processamento dos materiais}

Durante o processo de extrusão do PHB puro, constatou-se que houve uma alteração na coloração do polímero, passando da cor amarelada para um castanho escuro. Segundo Scott e Sharma ${ }^{[25,26]}$ este escurecimento pode ser resultado de resíduos bacteriais ou de solventes empregados no processo de extração. Porém, alguns estudos indicam que este comportamento possa ser resultante de um processo termodegradativo do polímero, sendo a intensidade de escurecimento proporcional ao grau de degradação, relacionado diretamente com a severidade das condições de processamento ${ }^{[8,27]}$. O processamento das blendas apresentou homogeneidade, produzindo um material com coloração castanho claro. A incorporação do pó de serra nas blendas apresentou dificuldades devido ao pequeno tamanho das partículas.

A injeção dos corpos de prova dos polímeros puros e de suas misturas foi satisfatória, permitindo a obtenção de corpos de prova homogêneos e com um acabamento superficial excelente.

\section{Avaliação macroscópica}

Para verificar a ocorrência de alterações macroscópicas nas amostras ao longo do tempo em contato com o solo, os corpos de prova das amostras foram fotografados.As Figuras 1 até 7 apresentam os corpos de prova das amostras em estudo antes do ensaio e 90 dias após o contato com o solo.

As Figuras 1 a 7 mostram que a degradação teve início a partir da adesão e crescimento de microrganismos na superfície do material, e que se intensifica com o passar do tempo. Qualitativamente verifica-se que as amostras que contém pó de serra são mais susceptíveis ao processo degradativo, embora seja possível verificar alterações na coloração para as amostras do copoliéster puro ao longo do tempo em contato com o solo.

Observou-se também nas Figuras 1 até 7 que o processo degradativo não é uniforme ao longo da superfície dos corpos de prova, variando também de um corpo de prova para o outro.

\section{Perda de massa ao longo da biodegradação}

Após serem submetidas ao teste de biodegradação em solo, as amostras foram lavadas manualmente, secadas a aproximadamente $50{ }^{\circ} \mathrm{C}$ por 72 horas em estufas com circulação de ar e pesadas para medir a perda de massa do material. A Tabela 1 apresenta os valores médios e a estimativa do desvio padrão para a perda de massa de cada material submetido ao teste, sendo que o teste foi realizado com cinco corpos de prova de cada material.

Através das análises de perda de massa, sugere-se que todos os materiais estudados são degradados por processos bióticos.

Analisando a Tabela 1 e a Figura 8 pode-se perceber que conforme aumenta o tempo em contato com o solo aumenta-se a quantidade de perda de massa do material, mas não de uma maneira

Tabela 1. Variação percentual da perda de massa das amostras estudadas para diferentes tempos em contato com o solo.

\begin{tabular}{|c|c|c|c|}
\hline Material & 30 dias $(\%)$ & 60 dias $(\%)$ & 90 dias $(\%)$ \\
\hline PHB & $0,23 \pm 0,12$ & $1,32 \pm 0,23$ & $19,17 \pm 3,43$ \\
\hline Eastar Bio ${ }^{\circledR}$ & $0,53 \pm 0,10$ & $1,44 \pm 0,06$ & $6,27 \pm 0,62$ \\
\hline PHB/Eastar Bio $^{\circledR}(75 / 25)$ & $0,17 \pm 0,01$ & $0,53 \pm 0,11$ & $2,83 \pm 0,23$ \\
\hline PHB/Ecoflex ${ }^{\circledR}(75 / 25)$ & $0,16 \pm 0,03$ & $0,38 \pm 0,09$ & $1,86 \pm 0,22$ \\
\hline PHB/Eastar $\mathrm{Bio}^{\circledR}(75 / 25)+30 \%$ p.d.s. & $0,29 \pm 0,11$ & $2,35 \pm 0,58$ & $29,32 \pm 4,58$ \\
\hline PHB/Ecoflex ${ }^{\circledR}(75 / 25)+30 \%$ p.d.s. & $0,40 \pm 0,05$ & $1,86 \pm 0,37$ & $13,98 \pm 1,83$ \\
\hline
\end{tabular}

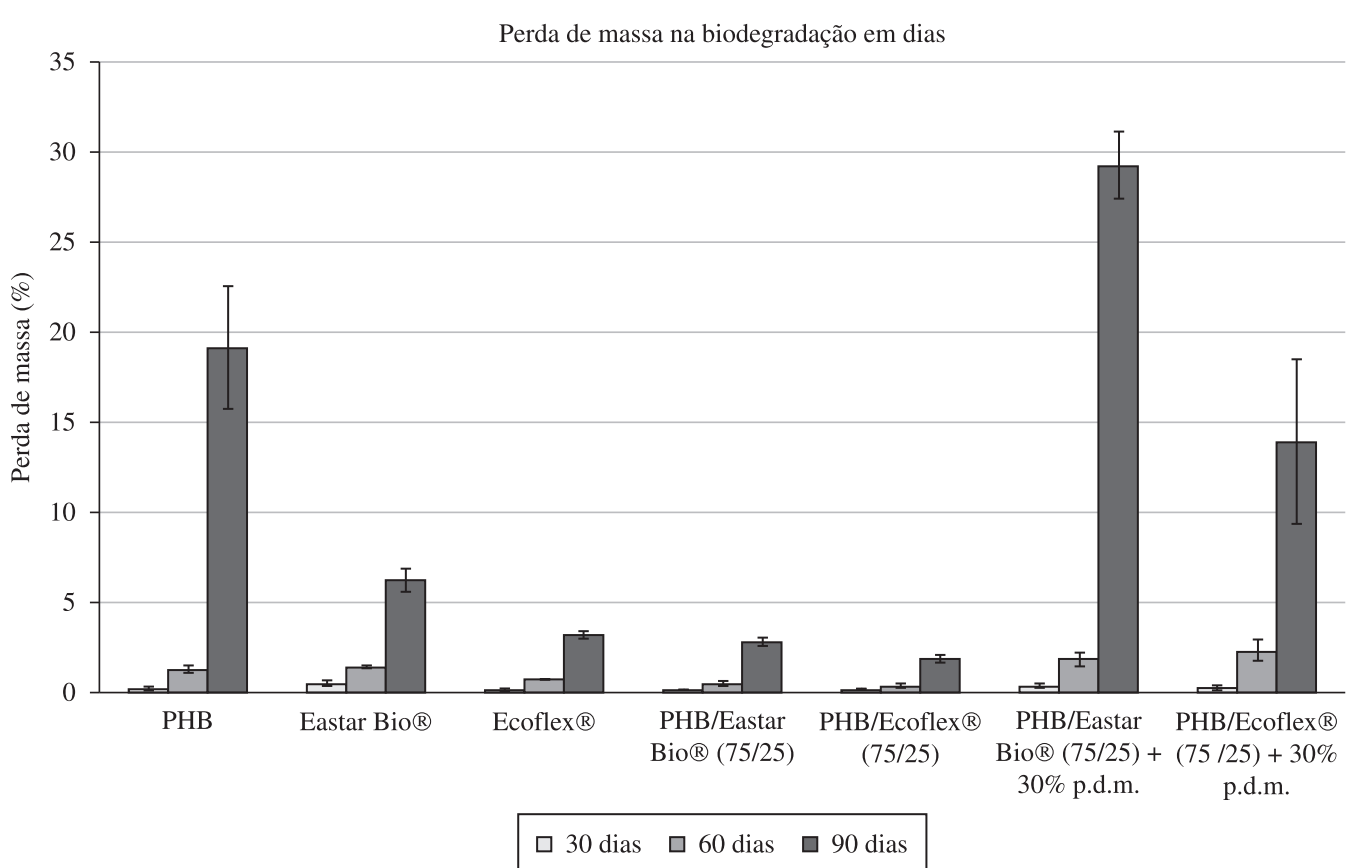

Figura 8. Variação percentual da perda de massa das amostras estudadas para diferentes tempos em contato com o solo. 
proporcional. Nota-se que as blendas com pó de serra possuem uma maior facilidade para perder massa na condição do teste.

\section{Alterações morfológicas das blendas ao longo da biodegradação}

Foi realizada a análise da morfologia na superfície da fratura criogênica das amostras antes e após 90 dias em contato com o solo. As Figuras 9 a 12 são referentes às micrografias das blendas antes e após 90 dias em contato com o solo.

Pode-se observar nas Figuras 9 a 12 que não há indícios de degradação no interior das amostras, tendo em vista a similaridade entre as morfologias apresentadas antes do contato com o solo e após 90 dias de ensaio.

Este resultado indica que provavelmente o processo degradativo ocorre através da erosão superficial dos corpos de prova.

\section{Resistência ao impacto das blendas ao longo da biodegradação}

Para verificar as propriedades mecânicas dos materiais após 30, 60 e 90 dias em contato com solo, foi realizado o ensaio mecânico de impacto, seguindo a norma ASTM D-256 ${ }^{[24]}$ e o procedimento já descrito.

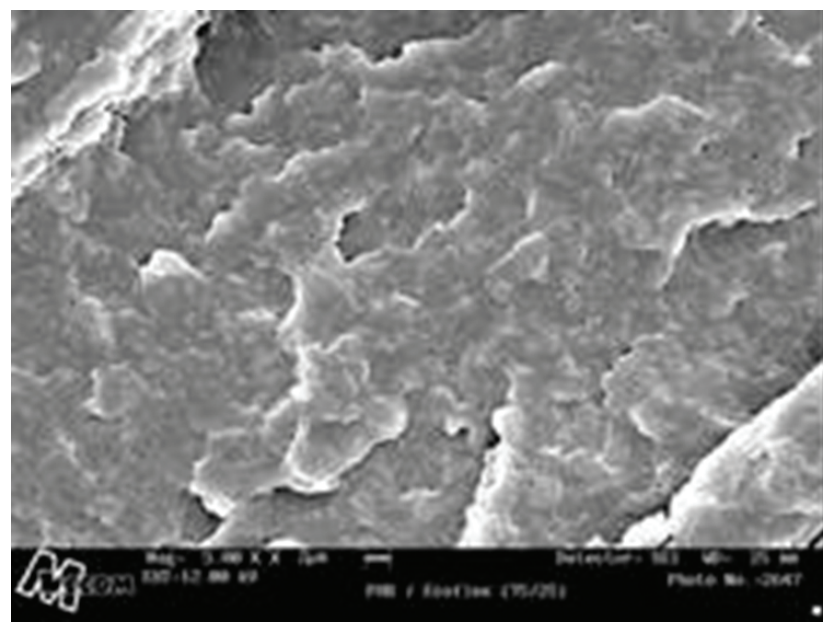

(a)
Para obter-se os resultados da resistência ao impacto foram ensaiados cinco corpos de prova para cada amostra, e com os valores obtidos foram calculados a média aritmética e a estimativa do desvio padrão. A Tabela 2 apresenta os resultados obtidos.

Através das análises de resistência ao impacto verificou-se que as misturas dos copoliésteres com o PHB apresentaram uma acentuada redução da rigidez na matriz de PHB.

Em relação à biodegradação o PHB puro apresentou maior perda de tenacidade em relação às blendas e aos compósitos, sendo que o PHB puro ao término de 90 dias teve uma redução de $23 \%$ de resistência ao impacto. A diminuição da tenacidade durante o processo de biodegradação ocorre devido à presença de vazios tanto na superfície quanto no interior dos corpos de prova, facilitando a propagação de trincas e diminuindo as propriedades mecânicas ${ }^{[29]}$. Das blendas modificadas com pó de serra estudadas observou-se que a blenda PHB/Eastar Bio $^{\circledR}$ (75/25)/pó de serra (70/30) perdeu mais massa ao longo do ensaio de biodegradação em solo, porém a blenda PHB/Ecoflex ${ }^{\circledR}(75 / 25) /$ pó de serra (70/30) apresentou maior redução de resistência ao impacto, em torno de $10 \%$ após 90 dias em contato com o solo, indicando que a perda de massa da blenda com o copoliéster Eastar Bio ${ }^{\circledR}$ foi superficial.

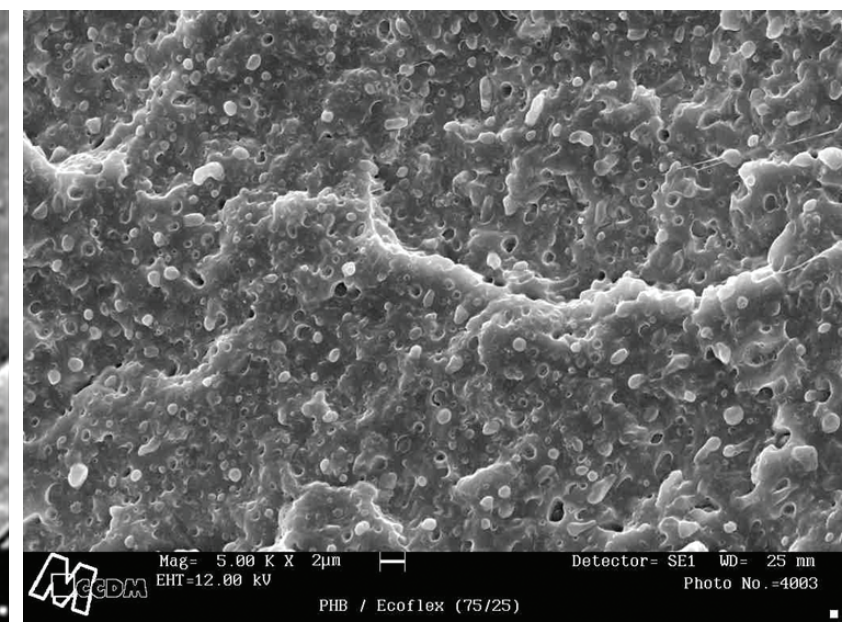

(b)

Figura 9. PHB/Ecoflex ${ }^{\circledR}(75 / 25)$ com um aumento de 5000× (a) antes e (b) após 90 dias de ensaio.

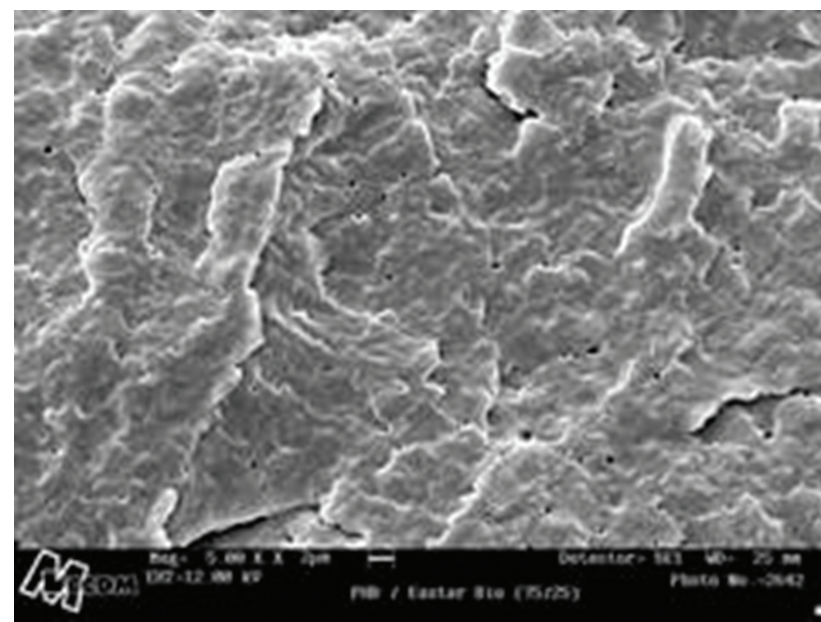

(a)

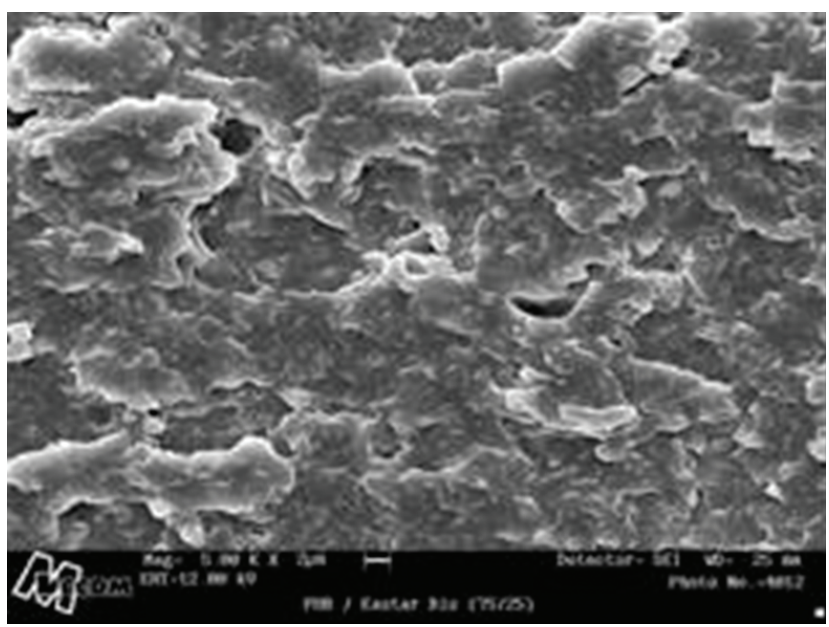

(b)

Figura 10. PHB/Eastar Bio $^{\circledR}(75 / 25)$ com um aumento de 5000× (a) antes e (b) após 90 dias de ensaio. 


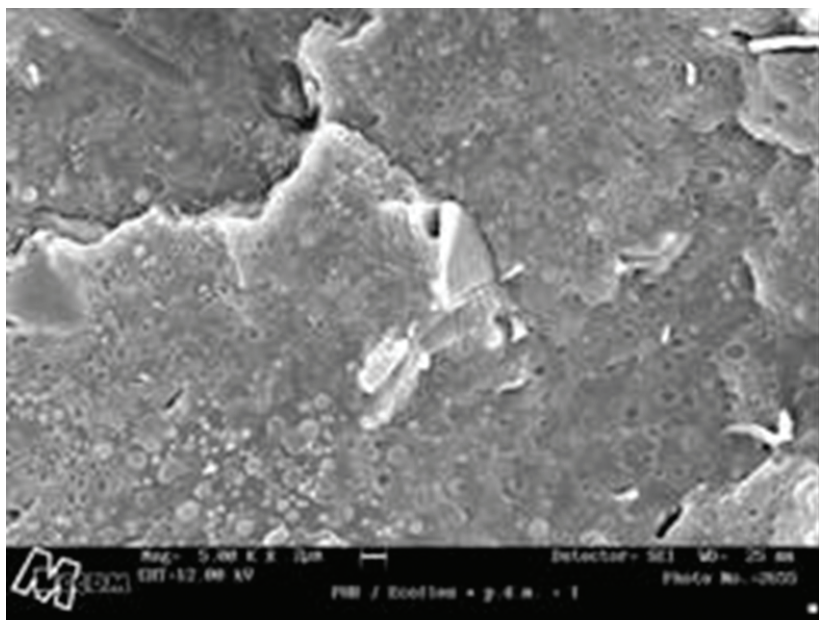

(a)

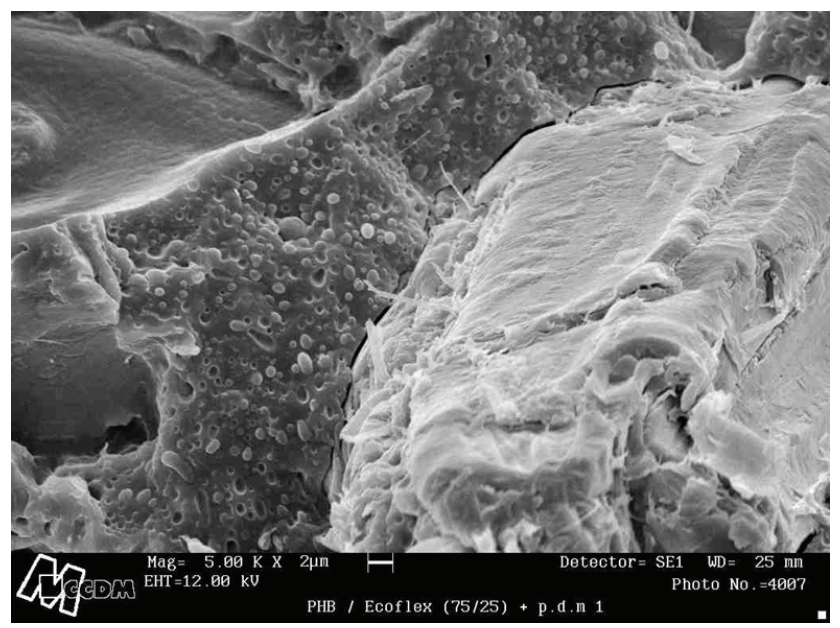

(b)

Figura 11. PHB/Ecoflex ${ }^{\circledR}(75 / 25)$ /pó de serra (70/30) com um aumento de 5000× (a) antes e (b) após 90 dias de ensaio.

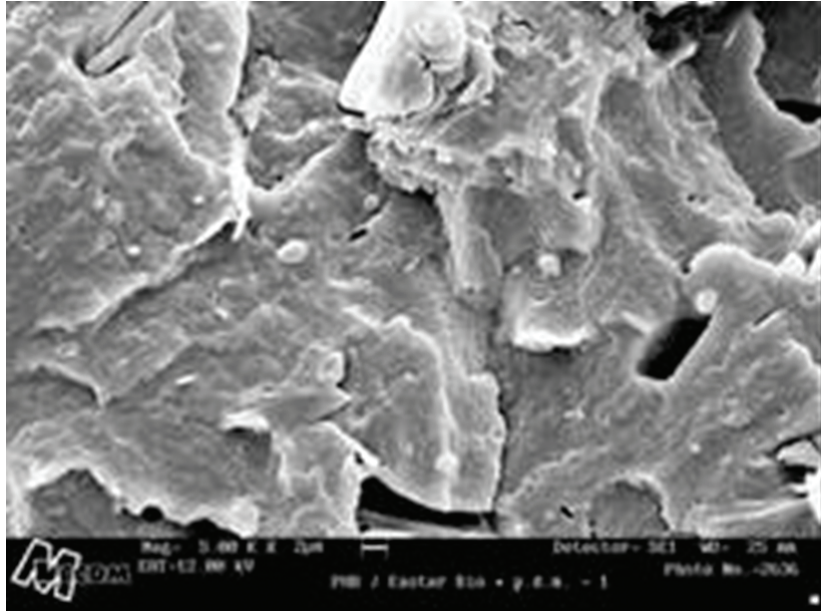

(a)

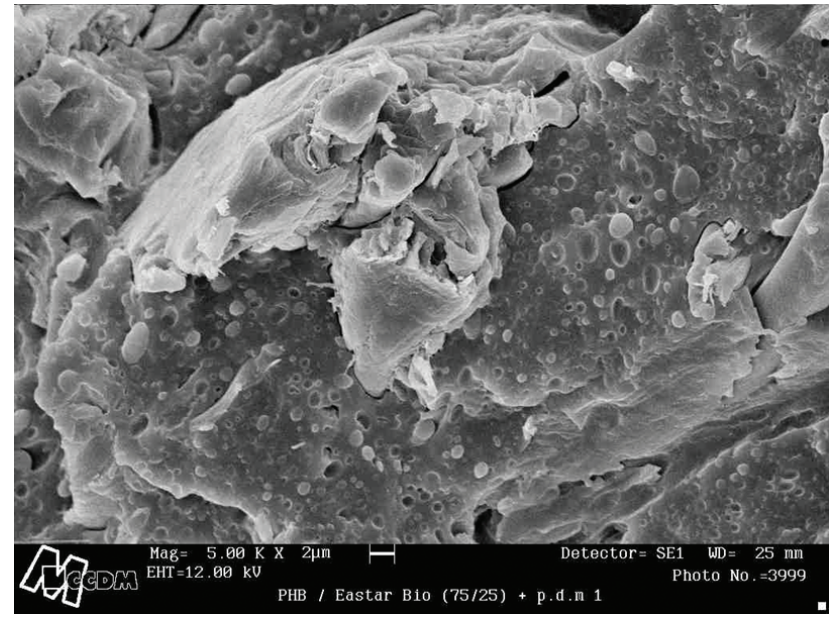

(b)

Figura 12. PHB/Eastar Bio $^{\circledR}(75 / 25)$ /pó de serra (70/30) com um aumento de 5000× (a) antes e (b) após 90 dias de ensaio.

Tabela 2. Resistência ao impacto (Izod, com entalhe) das blendas antes e ao longo da biodegradação.

\begin{tabular}{|c|c|c|c|c|}
\hline Materiais & Tempo zero $(\mathrm{J} / \mathbf{m})$ & $30 \operatorname{dias}(\mathrm{J} / \mathrm{m})$ & $60 \operatorname{dias}(\mathrm{J} / \mathrm{m})$ & $90 \operatorname{dias}(\mathrm{J} / \mathrm{m})$ \\
\hline PHB & $27,0 \pm 0,0$ & $22,1 \pm 0,0$ & $21,6 \pm 0,0$ & $20,6 \pm 0,0$ \\
\hline PHB/Eastar Bio ${ }^{\circledast}(75 / 25)$ & $23,6 \pm 0,5$ & $26,4 \pm 0,0$ & $22,1 \pm 0,0$ & $26,2 \pm 0,0$ \\
\hline PHB/Ecoflex ${ }^{\circledR}(75 / 25)$ & $22,3 \pm 0,5$ & $22,6 \pm 0,0$ & $20,9 \pm 0,0$ & $22,9 \pm 0,0$ \\
\hline PHB/Eastar $\mathrm{Bio}^{\circledast}(75 / 25)+30 \%$ p.d.s. & $23,6 \pm 0,0$ & $20,9 \pm 0,0$ & $21,4 \pm 0,0$ & $22,6 \pm 0,0$ \\
\hline PHB/Ecoflex ${ }^{\otimes}(75 / 25)+30 \%$ p.d.s. & $26,7 \pm 0,0$ & $23,1 \pm 0,0$ & $21,6 \pm 0,0$ & $23,9 \pm 0,0$ \\
\hline
\end{tabular}

\section{Conclusões}

Observou-se que os três polímeros biodegradaram em contato com o solo, assim como as blendas PHB/Ecoflex ${ }^{\circledR}(75 / 25)$, PHB/Eastar Bio $^{\circledR}(75 / 25)$, PHB/Ecoflex ${ }^{\circledR}(75 / 25) /$ pó de serra (70/30) e PHB/Eastar Bio ${ }^{\circledR}(75 / 25) /$ pó de serra (70/30). Verificou-se que as blendas modificadas com pó de serra e o polímero PHB puro foram os materiais que perderam mais massa durante os 90 dias de teste de biodegradação em solo e apresentaram maior redução de resistência ao impacto. Um aspecto importante a ser considerado é que a incorporação de $30 \%$ de pó de madeira, em massa, na mistura $\mathrm{PHB} /$ copoliésteres, apresenta grande vantagem econômica devido à redução do custo do material.

Concluiu-se também que a biodegradação de todos os materiais estudados iniciam-se pela superfície, e que o tempo de 90 dias é insuficiente para alterar a morfologia interna dos materiais.

\section{Agradecimentos}

À FAPESP - Fundação de Amparo à Pesquisa do Estado de São Paulo pela bolsa, à PHB Industrial S/A pelo fornecimento dos polímeros, à Pinhopó Moagem de Madeiras Ltda pelo fornecimento do pó de serra, à Provaso Indústria e Comércio de Fertilizantes Orgânicos Ltda pelo fornecimento do solo (composto orgânico) e ao PPG-CEM - UFSCar pela oportunidade de realização do Mestrado.

\section{Referências Bibliográficas}

1. Pantano Filho, R.; Rosa, D. S.; Chui, Q. S. H. \& Agnelli, J. A. M. - "Avaliação da biodegradação de PHB, PHB-V e PCL em solo compostado", in: Anais do $6^{\circ}$ Congresso Brasileiro de Polímeros, Belo Horizonte - MG, (2001).

2. Faria, A. U. \& Franchetti, S. M. M. - Polímeros, 20, p.141 (2010). http://dx.doi.org/10.1590/S0104-14282010005000024 
3. Chiellini, E. \& Solaro, R. - "Environmentally Degradable Polymers and Plastics. An Overview", in: Proceedings of International Workshop on Environmentally Degradable and Recyclable Polymers in Latin American, p.15, Campinas - SP, (1998).

4. Duarte, M. A. T.; Júlio, C. F.; Martins, E. S. \& Pezzin, S. H. - "Estudo da compactação a frio de poli(3-hidroxibutirato) - PHB morfologia e resistência à compressão", in: Anais do $1^{\circ}$ Congresso em Ciência de Materiais do Mercosul, Joinville - SC, (2004).

5. Rosa, D. S. \& Carraro, G. - "Avaliação de plásticos biodegradáveis sob envelhecimento acelerado em solo com diferentes $p H$ ", in: Anais do $5^{\circ}$ Congresso Brasileiro De Polímeros, p.274, Águas de Lindóia - SP, (1999).

6. Pellicano, M.; Pachekoski, W. \& Agnelli, J.A.M. - Polímeros, 19, p.212 (2009). http://dx.doi.org/10.1590/S0104-14282009000300009

7. Machado, M. L. C.; Pereira, N. C.; Miranda, L. F. \& Terence, M. C. - Polímeros, 20, p.65 (2010). http://dx.doi.org/10.1590/S010414282010005000011

8. Pachekoski, W. M. - "Desenvolvimento de blendas poliméricas do polímero biodegradável poli-hidroxibutirato $(\mathrm{PHB})$ com polipropileno $(P P)^{\prime}$, Dissertação de Mestrado, Universidade Federal de São Carlos, Brasil (2001).

9. Yoshie, N.; Saito, M. \& Inoue, Y. - Polymer, 45, p.1903 (2004). http:// dx.doi.org/10.1016/j.polymer.2004.01.025

10. Muller, R.; Kleeberg, I. \& Deckwer, W. - J. Biotechnol., 86, p.87 (2001). http://dx.doi.org/10.1016/S0168-1656(00)00407-7

11. Wang, L.; Ma, W.; Gross, R. A. \& McCarthy, S. P. - Polym. Degrad. Stabil., 59, p.1161 (1998).

12. Zhang, L.; Xiong, C. \& Deng, X. - Polymer, 37, p.235 (1996). http:// dx.doi.org/10.1016/0032-3861(96)81093-7

13. Kopinke, F. D.; Remmler, M; Mackenzie, K.; Möder, M. \& Wachsen, O. - Polym. Degrad. Stabil., 53, p.329 (1996). http://dx.doi. org/10.1016/0141-3910(96)00102-4

14. Witt, U.; Einig, T.; Yamamoto, M.; Kleeberg, I.; Deckwer W.-D. \& Müller, R.-J. - Chemosphere, 44, p.289 (2001). http://dx.doi org/10.1016/S0045-6535(00)00162-4

15. Mani, R. \& Mrinal, B. - Eur. Polym. J., 37, p.515 (2001). http://dx.doi. org/10.1016/S0014-3057(00)00155-5
16. Fang, Q. \& Hanna, M. A. - Bioresour. Technol., 78, p.115 (2001). http://dx.doi.org/10.1016/S0960-8524(01)00013-X

17. Squio, C. R. \& Aragão, G. M. F. - Quim. Nova, 27, p.615 (2004). http:// dx.doi.org/10.1590/S0100-40422004000400016

18. Velho, L. \& Velho, P. - "Resumos da Technology Transfer Society Conference", Atlanta, Georgia, 2006.

19. Quental, A. C.; Carvalho, F. P., Tada, E. S. \& Felisberti, M. I.Quim. Nova, 33, p.438 (2010). http://dx.doi.org/10.1590/S010040422010000200035

20. Oliveira, M. "Por que o plástico demora tanto tempo para desaparecer na natureza?", O Florense. 2009

21. Raupp, A. - "Embalagem Marca", p.57 (2007).

22. American Society for Testing and Materials - ASTM. - "D 6003-96: Standard Test Method for Determining Weight Loss from Plastic Materials Exposed to Simulated Municipal Solid-Wast (MSW) Aerobic Compost Environment”, Annual Book of ASTM Standards, Philadelphia (1996).

23. American Society for Testing and Materials - ASTM. - "G 160-98 "Standard Practice for Evaluating Microbial Susceptility of Nonmetallic Materials by Laboratory Soil Burial", Annual Book of ASTM Standard, 1998

24. American Society for Testing and Materials - ASTM. - "D256-02 "Standard Test Methods Determining the Pendulum Impact of Notched Specimens of Plastics", Annual Book of ASTM Standards, Philadelphia (2002).

25. Scott, G. \& Gillead, D. - "Degradable Polymers", Chapman \& Hall, London, 1995. http://dx.doi.org/10.1007/978-94-011-0571-2

26. Sharma, R. \& Ray, A. R. J.M.S. - Rev. Macromol. Chem. Phys., C35, p.327 (1995). http://dx.doi.org/10.1080/15321799508009640

27. Rosário, F. - "Estudo das modificações do PHB com PCL, com farinha de madeira e com plastificantes", Tese Doutorado, Universidade Federal de São Calos, Brasil (2005).

28. Luo, S. \& Netravali, A. N. - Polym. Degrad. Stab., 80, p.59 (2003). http://dx.doi.org/10.1016/S0141-3910(02)00383-X

Enviado: $23 / 02 / 11$

Reenviado: $20 / 01 / 12$

Aceito: $23 / 03 / 12$ 